\title{
Avaliação da qualidade do pré-natal a partir das recomendações do Programa de Humanização no Pré-natal e Nascimento
}

\author{
Evaluation of the quality of prenatal care based on the \\ recommendations Prenatal and Birth Humanization Program
}

Rosemar Barbosa Mendes (https://orcid.org/0000-0002-4860-7413) ${ }^{1}$

José Marcos de Jesus Santos (https://orcid.org/0000-0001-5122-1469) ${ }^{2}$

Daniela Siqueira Prado (https://orcid.org/0000-0002-3901-3832) ${ }^{3}$

Rosana Queiroz Gurgel (https://orcid.org/0000-0001-6241-0134) ${ }^{4}$

Felipa Daiana Bezerra (https://orcid.org/0000-0002-5328-2350) ${ }^{5}$

Ricardo Queiroz Gurgel (https://orcid.org/0000-0001-9651-3713) ${ }^{3}$

\footnotetext{
${ }^{1}$ Departamento de Enfermagem, Universidade Federal de Sergipe. Av. Marechal Rondon s/n, Jardim Rosa Elze. 49100-000 São Cristóvão SE Brasil. rosemarbm@uol.com.br ${ }^{2}$ Programa de PósGraduação Enfermagem em Saúde Pública, Escola de Enfermagem de Ribeirão Preto, Universidade de São Paulo. Ribeirão Preto SP Brasil.

${ }^{3}$ Departamento de Medicina, Universidade Federal de Sergipe. São Cristóvão SE Brasil. ${ }^{4}$ Departamento de Enfermagem, Universidade Tiradentes Aracaju SE Brasil.

${ }^{5}$ Departamento de Enfermagem, Centro Universitário Estácio de Sergipe. Aracaju SE Brasil.
}

\begin{abstract}
The Prenatal Care and Birth Humanization Program (PHPN) establishes a minimum number of procedures to be provided to all pregnant women during prenatal care. This study aimed to analyze the quality of prenatal care in Sergipe based on the PHPN recommendations. This is a cross-sectional study, with a descriptive and analytic approach, using survey data from the Birth in Sergipe research, conducted from June 2015 to April 2016 with 768 puerperae proportionally distributed among all state maternity hospitals $(n=11)$. Data were collected from face-to-face interviews and patients' prenatal care cards. The results showed a high coverage of prenatal care (99.3\%; $n=763)$, but little more than half of these women started their prenatal care within 16 weeks of gestation (57\%; $n=435)$, and $74.7 \%(n=570)$ had six or more visits. We noted that $16.6 \%(n=127)$ of pregnant women were at high risk for complications and almost half continued monitoring prenatal care with professional nurses. Around $61.3 \%$ were advised about the maternity care service of reference for delivery, and $29.4 \%$ sought more than one health service for childbirth. We concluded that there was a high prenatal care coverage in Sergipe, however, with issues concerning its adaptation to the PHPN.
\end{abstract}

Key words Pregnant Women, Prenatal care, Women's Health
Resumo O Programa de Humanização no Prénatal e Nascimento (PHPN) estabelece um pacote mínimo de procedimentos a serem oferecidos a todas as gestantes durante o pré-natal. O objetivo deste estudo foi analisar a qualidade do pré-natal no estado de Sergipe a partir das recomendações do PHPN. Trata-se de um estudo transversal, com abordagens descritiva e analítica, vinculado à pesquisa Nascer em Sergipe, realizada entre junho/2015 e abril/2016. Foram avaliadas 768 puérperas proporcionalmente distribuidas entre todas as maternidades do estado $(n=11)$. A coleta dos dados ocorreu por meio de entrevista face a face $e$ visualização do cartão de pré-natal. Os resultados mostraram uma cobertura elevada da assistência pré-natal (99,3\%; $n=763)$, porém pouco mais da metade destas mulheres iniciaram seu acompanhamento antes da $16^{a}$ semana gestacional (57\%; $n=435)$ e $74,4 \%(n=570)$ compareceram a seis ou mais consultas. Constatou-se que 16,6\% (n $=127$ ) das gestantes foram consideradas de alto risco e quase metade delas continuou o acompanhamento com profissionais enfermeiros. A orientação sobre a maternidade de referência para o parto foi referida por $61,3 \%$ e 29,4\% procuraram mais de um serviço para a parturição. Concluiuse que houve uma alta cobertura do pré-natal em Sergipe, porém com problemas relacionados à sua adequação ao PHPN.

Palavras-chave Gestantes, Cuidado Pré-Natal, Saúde da Mulher 


\section{Introdução}

A assistência pré-natal inclui um conjunto de medidas que visam levar a partos de recém-nascidos saudáveis, sem impactos negativos na saúde das mulheres, com abordagem inclusive dos aspectos psicossociais e das atividades educativas e preventivas cabíveis neste processo ${ }^{1}$. Inclui ações de promoção e prevenção da saúde, além de diagnóstico e tratamento adequado dos problemas que possam vir a ocorrer ${ }^{2}$, sendo, portanto, eficaz na redução da morbimortalidade relacionada ao ciclo gravídico-puerperal para as mães e seus recém-nascidos ${ }^{3}$.

Nesse sentido, o Ministério da Saúde por meio do Programa de Humanização no Pré-natal e Nascimento (PHPN) ${ }^{4}$ estabelece um pacote mínimo de procedimentos e exames a serem oferecidos à todas as gestantes durante a atenção pré-natal: (a) início da assistência até o quarto mês de gestação (16a semana); (b) mínimo de seis consultas, preferencialmente uma no primeiro trimestre gestacional, duas no segundo e três no terceiro; (c) rotina de exames laboratoriais e vacinação, (d) atividades educativas e (e) consulta puerperal.

Vale ressaltar que a assistência pré-natal, muitas vezes, representa o primeiro contato das mulheres com os serviços de saúde, e por isso deve ser organizada de forma a atender suas reais necessidades, por meio da utilização de conhecimentos técnico-científicos e dos recursos preconizados ${ }^{5}$, num contexto de humanização ${ }^{2}$. Todavia, estudos têm identificado falhas que interferem na sua qualidade e efetividade, tais como baixa cobertura $^{6}$, início tardio, distribuição inadequada das consultas $^{7}$ ou em número insuficiente, realização incompleta dos procedimentos preconizados ${ }^{8} \mathrm{e}$ carência de informações sobre a maternidade de referência para o parto 9 .

Observa-se ainda a escassez de trabalhos nacionais que avaliam a assistência pré-natal à nível estadual. No Sergipe, as informações do Sistema de Informações sobre Nascidos Vivos (SINASC) demonstram que há grande acesso à atenção pré-natal, mas apenas pouco mais da metade das gestantes $(52,1 \%)$ realizaram mais de seis consultas de pré-natal em $2014^{10}$. Entretanto, como observado por Viellas et al. ${ }^{9}$, esse sistema só permite a análise do número de consultas realizadas, além de se restringir às gestações que resultaram num nascido vivo. De igual modo, o Sistema de Acompanhamento do Programa de Humanização no Pré-Natal e Nascimento (SISPRENATAL), software desenvolvido para fornecer informações fundamentais para planejamento, acompanhamento e avaliação das ações desenvolvidas pelo PHPN, apresenta problemas de subnotificação, com falhas de registro dos procedimentos mínimos recomendados pelo Programa na atenção as gestantes, em comparação com outras fontes de informação ${ }^{11}$.

Desta forma, a pouca disponibilidade de dados sobre a assistência materno-infantil do estado apresenta-se como importante entrave para sua avaliação e planejamento, além de impossibilitar a implementação de medidas eficazes e resolutivas. Assim, objetivou-se analisar a qualidade da assistência pré-natal oferecida às usuárias de serviços de saúde públicos e/ou privados de Sergipe, a partir das recomendações do $\mathrm{PHPN}^{4}$, e sua forma de organização com os serviços de saúde materno-infantil do estado.

\section{Métodos}

Trata-se de um estudo transversal e quantitativo, com abordagens descritiva e analítica, vinculado à pesquisa Nascer em Sergipe, realizada entre junho de 2015 e abril de 2016. Foram avaliadas 768 puérperas e seus conceptos proporcionalmente distribuídos entre as maternidades públicas, privadas e mistas do estado de Sergipe $(\mathrm{n}=$ 11). Reproduziu-se o método da pesquisa Nascer no Brasil ${ }^{12}$, com treinamento da equipe local por pesquisadoras da Fundação Oswaldo Cruz (Fiocruz) que participaram do estudo nacional.

O cálculo amostral, com nível de confiança de $95 \%$, teve um desenho probabilístico, em dois estágios. O primeiro correspondeu aos estabelecimentos de saúde e o segundo às puérperas. Foram elegíveis todas as maternidades do estado que registraram no mínimo 500 partos/ano, totalizando-se sete instituições no interior e quatro na capital, sendo cinco públicas, quatro mistas e duas privadas. As puérperas foram selecionadas por amostragem aleatória simples, a partir de uma listagem de internação diária, onde foram consideradas elegíveis todas as mulheres com parto de feto vivo e parto de feto morto com peso ao nascer maior ou igual a $500 \mathrm{~g}$ e/ou idade gestacional maior ou igual a 22 semanas (dados obtidos por meio de consulta aos prontuários dos recém-nascidos e parturientes). Foram excluídas mulheres que não falassem e/ou compreendessem o idioma (português) e que apresentassem transtornos mentais graves. Ressalta-se que foi adotada alocação proporcional ao tamanho da instituição para distribuir o número amostral calculado $(\mathrm{n}=768)$. 
Os entrevistadores permaneceram no mínimo 7 dias em cada instituição. Caso o número de puérperas pudesse ser atingido antes deste período, procedia-se a um sorteio aleatório com a limitação do número diário de entrevistadas, para que os 7 dias fossem atingidos. Foram realizadas entrevistas face a face com as puérperas com intervalo mínimo de 6hs após o parto e extraídos dados do prontuário da mulher e do recém-nascido após a alta (ou óbito). Os cartões de pré-natal, com autorização das participantes, foram fotografados e as informações digitadas no banco de dados. Informação mais detalhada sobre a coleta é também relatada em Leal et al. ${ }^{13}$.

As variáveis estudadas para avaliação da assistência pré-natal foram as constantes no PHPN: cobertura do pré-natal (realização de pelo menos uma consulta); início precoce (até a 16 a semana gestacional); número de consultas realizadas; recebimento do cartão de pré-natal; exames registrados no cartão (glicemia e ultrassonografia) e recebimento de informações durante este processo (conhecimento sobre o trabalho de parto, sinais de risco na gravidez e aleitamento materno). Para as mulheres que apresentaram cartão da gestante $(n=599)$, considerou-se a idade gestacional na primeira consulta de pré-natal e o total de consultas registradas. Para as mulheres que não apresentaram cartão $(n=169)$, utilizou-se a informação obtida durante a entrevista ${ }^{14}$.

As variáveis sociodemográficas maternas investigadas foram: idade, raça/cor da pele, nível de escolaridade, trabalho remunerado, situação conjugal e local de moradia. Investigou-se também o planejamento da gravidez, a satisfação da mulher ao descobri-la e tentativas abortivas.

$\mathrm{Na}$ análise das variáveis relacionadas ao prénatal, foi considerada a frequência de mulheres que realizaram esse acompanhamento $(n=763)$, sendo avaliadas associações deste processo com as características sociodemográficas e gestacionais.

Também foi verificada a forma de estruturação dos serviços de pré-natal segundo fonte de pagamento (público ou privado), tipo de unidade de saúde onde a mulher realizou a maioria das consultas de pré-natal (atenção básica ou ambulatório hospitalar), profissional que prestou a assistência pré-natal na maioria das consultas (médico ou enfermeiro) e continuidade da assistência (mesmo profissional durante toda a assistência pré-natal ou não). As orientações oferecidas às mulheres durante o pré-natal foram avaliadas segundo o número de consultas realizadas ( $\leq 5$ consultas ou $\geq 6$ consultas).
A integração da assistência pré-natal a outros serviços da rede de saúde foi aferida pelas seguintes proporções: gestantes consideradas de risco encaminhadas e atendidas em serviços de referência; gestantes orientadas sobre o local de internação para o parto; gestantes que foram atendidas nesses serviços e necessidade de busca de mais de um serviço no momento da internação para o parto (denominada "peregrinação").

Variáveis relacionadas ao planejamento da gestação atual (queria/não queria engravidar/ queria esperar mais tempo) e à satisfação da gestante com essa gravidez (satisfeita/mais ou menos satisfeita/ insatisfeita) também foram utilizadas para análise dos desfechos "cobertura do pré-natal" e "início precoce".

Adotou-se a convenção elaborada pela Organização Mundial da Saúde para determinação da faixa etária, na qual a adolescência é delimitada de 10 a 19 anos $^{15}$.

Para a análise estatística, foram utilizadas as técnicas univariada e bivariada para obtenção da distribuição dos valores das frequências absoluta e relativa. O teste Qui-quadrado de independência de Pearson foi utilizado para avaliar as associações entre as variáveis categóricas com significância $<0,05$. O pacote utilizado foi o IBM ${ }^{\circledast}$ SPSS - Statistical Package for the Social Sciences, versão 20.0.

Este estudo foi aprovado pelo Comitê de Ética em Pesquisa da Universidade Federal de Sergipe. Todos os cuidados foram adotados visando garantir o sigilo e confidencialidade das informações, conforme Resolução no 466/2012 ${ }^{16}$, que atualiza a resolução no $196 / 1996^{17}$ do Conselho Nacional de Saúde do Ministério da Saúde, Brasília, DF. As puérperas assinaram o Termo de Consentimento Livre e Esclarecido (TCLE), com garantia de recusa a qualquer momento, sem sofrer danos.

\section{Resultados}

Participaram do estudo 768 puérperas cuja média de idade era de 25,3 $\pm 6,5$ anos, sendo $21,4 \%$ $(\mathrm{n}=164)$ adolescentes e $9,4 \%(\mathrm{n}=72)$ com idade $\geq 35$ anos. A raça/cor da pele parda foi a mais referida $(75 \% ; \mathrm{n}=576)$, com amarelas e indígenas correspondendo a uma proporção pequena do total da amostra (3\% e 0,4\%, respectivamente). Quanto à escolaridade, 53,6\% $(\mathrm{n}=412)$ possuíam ensino médio ou superior. Mais da metade não trabalhava de forma remunerada $(55,5 \%$; $\mathrm{n}$ $=426)$, vivia com o companheiro $(63,2 \% ; \mathrm{n}=$ 
485) e morava na capital sergipana $(57,8 \%$; $\mathrm{n}=$ 444). A gravidez planejada foi referida por $41,1 \%$ $(\mathrm{n}=316)$ e $35,9 \%(\mathrm{n}=276)$ sentiram-se insatisfeitas ao descobri-la. Tentativas abortivas foram relatadas por $4,9 \%$ das mulheres entrevistadas (n = 38) (dados não apresentados em tabela).

A cobertura da assistência pré-natal em Sergipe foi de $99,3 \%$, sendo sempre superior a $90 \%$ independente da característica materna. Os menores percentuais estão relacionados às adolescentes, às que não queriam engravidar, às insatisfeitas com a gestação e que referiram tentativas abortivas $(\mathrm{p}<0,05)$ (Tabela 1$)$.

Mais da metade das gestantes que realizaram o pré-natal iniciaram esse acompanhamento antes da $16^{\mathrm{a}}$ semana gestacional $(57 \% ; \mathrm{n}=435)$, sobretudo entre as que possuíam ensino médio, com companheiro, que desejavam engravidar, satisfeitas com a gravidez e que não tentaram interrompê-la $(\mathrm{p}<0,05)$. Em relação ao número de consultas, $74,7 \%(\mathrm{n}=570)$ tiveram as seis consultas mínimas preconizadas pelo Ministério da Saúde, principalmente entre as mulheres com maior escolaridade, primigestas ou secundigestas, que desejavam engravidar e que se sentiram satisfeitas ao descobri-la $(\mathrm{p}<0,05)$. Vale ressaltar que a proporção de mulheres com início precoce do pré-natal e com número suficiente de consultas não se mostrou similar à observada para a cobertura pré-natal (Tabela 1).

O recebimento do cartão de pré-natal foi mencionado por $96,6 \%(n=733)$ das entrevistadas e $81,7 \%(n=599)$ delas o apresentaram na admissão hospitalar para o parto. A maioria dos cartões analisados apresentavam registro do resultado da primeira glicemia $(85 \% ; \mathrm{n}=504)$ e da primeira ultrassonografia $(88,3 \% ; n=529)$, sendo o resultado da segunda glicemia observado em 53,6\% ( $n=270)$ dos que possuíam a primeira (dados não apresentados em tabela).

Quanto às orientações durante o pré-natal, $37,4 \%(n=275)$ das mulheres foram informadas sobre as atividades para facilitar o parto e $43,5 \%$ $(\mathrm{n}=320)$ dos sinais de início do trabalho de parto. Do mesmo modo, pouco mais da metade das mulheres receberam informações das alterações clínicas que indicariam risco da gravidez (56,9\%; $\mathrm{n}=419)$ e da importância da amamentação na primeira hora de vida do recém-nascido $(59,6 \%$; $\mathrm{n}=439$ ) (Tabela 2).

Pontua-se que as orientações sobre os sinais de início do trabalho de parto, alterações clínicas que indicariam risco da gravidez e importância da amamentação na primeira hora de vida foram mais frequentes entre as mulheres que realizaram 6 ou mais consultas de pré-natal $(\mathrm{p}<0,05)$ (Tabela 2).

A maioria das consultas foi realizada em serviços públicos $(71,6 \% ; n=546)$, principalmente por mulheres de menor idade e escolaridade e com companheiro $(\mathrm{p}<0,05)$. Estas consultas aconteceram, sobretudo, em unidades de atenção básica $(64,9 \% ; n=495)(\mathrm{p}<0,05)$. No que se refere ao profissional com quem a gestante realizou as consultas do pré-natal, não foi observada diferença significativa entre as categorias, já que $49,4 \%(\mathrm{n}=377)$ das mulheres foram acompanhadas por médicos e $48 \%(\mathrm{n}=366)$ por enfermeiros (Tabela 3).

Ao analisar a continuidade da assistência prénatal, constatou-se que $11,9 \%(\mathrm{n}=91)$ das mulheres referiram não terem sido acompanhadas pelo mesmo profissional ao longo da gestação. A maior proporção de continuidade com o mesmo profissional foi identificada em mulheres com maior idade ( $\mathrm{p}<0,05)$ (Tabela 3$)$.

Quanto ao risco gestacional, 16,6\% $(n=127)$ das puérperas referiram que a gestação foi classificada como de alto risco obstétrico, principalmente entre àquelas que pertenciam aos extremos de idade $(\mathrm{p}<0,05)$. Destas, 55,1\% $(\mathrm{n}=70)$ foram encaminhadas para serviços de referência e, apesar de a maioria ter referido a obtenção do atendimento, $21,4 \%(\mathrm{n}=15)$ afirmaram que o conseguiram com dificuldade (Tabela 4 ).

Mais da metade $(61,3 \% ; n=468)$ receberam informações sobre a maternidade de referência para internação para o parto. Mulheres com idade entre 15 e 19 anos, com companheiro e menor escolaridade foram as que menos relataram terem recebido essa orientação $(\mathrm{p}<0,05)$ (Tabela 4). Dentre as que foram orientadas, $83,3 \%(\mathrm{n}=$ 390) tiveram o seu parto assistido na maternidade indicada (dados não apresentados em tabela).

Antes de conseguir internação onde o parto foi realizado, 29,4\% $(\mathrm{n}=226)$ das mulheres buscaram assistência em outra maternidade, principalmente entre as que possuem menor idade e escolaridade $(\mathrm{p}<0,05)$ (Tabela 4$)$. A maioria dessas mulheres procurou apenas um serviço antes do atual $(87,6 \% ; n=198)$, porém $12,4 \%(n=$ 28) referiram ter procurado duas ou três unidades antes de conseguir a internação para o parto (dados não apresentados em tabela).

O Gráfico 1 apresenta as principais inadequações da assistência pré-natal no estado de Sergipe em relação às recomendações do PHPN. 
Tabela 1. Distribuição proporcional das características maternas segundo a cobertura da assistência pré-natal $(\mathrm{n}=768)$, início precoce, recebimento do cartão da gestante e número de consultas realizadas $(\mathrm{n}=763)$. Sergipe, Brasil, 2015/2016

\begin{tabular}{|c|c|c|c|c|c|c|c|c|c|c|}
\hline \multirow[t]{2}{*}{ Exposição/Desfecho } & \multirow[t]{2}{*}{$\begin{array}{c}\text { Assistência } \\
\text { pré-natal }\end{array}$} & \multirow[t]{2}{*}{$\mathrm{p}$} & \multirow[t]{2}{*}{$\begin{array}{l}\text { Início } \\
\text { precoce }\end{array}$} & \multirow[t]{2}{*}{$\mathbf{p}$} & \multirow{2}{*}{$\begin{array}{c}\text { Recebeu } \\
\text { o cartão } \\
\text { de pré- } \\
\text { natal }\end{array}$} & \multirow[t]{2}{*}{$\mathbf{p}$} & \multicolumn{3}{|c|}{$\begin{array}{c}\text { Número de } \\
\text { consultas de pré- } \\
\text { natal }\end{array}$} & \multirow[t]{2}{*}{$\mathbf{p}$} \\
\hline & & & & & & & $1-3$ & 4-5 & $\geq 6$ & \\
\hline Município & $\%$ & & $\%$ & & $\%$ & & $\%$ & $\%$ & $\%$ & \\
\hline Aracaju & 99,1 & & 54,3 & & 93,7 & & 4,5 & 15,3 & 80,2 & \\
\hline Capela & 100 & & 51,7 & & 100 & & 3,4 & 13,8 & 82,8 & \\
\hline Estância & 100 & & 66,1 & & 100 & & 9,7 & 27,4 & 62,9 & \\
\hline Glória & 100 & & 45,5 & & 100 & & 9,1 & 18,2 & 72,7 & \\
\hline Itabaiana & 98,8 & 0,939 & 69,8 & 0,085 & 97,7 & 0,265 & 4,7 & 12,8 & 82,6 & 0,058 \\
\hline Lagarto & 100 & & 50 & & 100 & & 3 & 15,2 & 81,8 & \\
\hline Propriá & 100 & & 62,9 & & 100 & & 2,9 & 25,7 & 71,4 & \\
\hline Socorro & 100 & & 54,2 & & 95,8 & & 16,7 & 25 & 58,3 & \\
\hline \multicolumn{11}{|l|}{ Idade (anos) } \\
\hline $10-14$ & 92,9 & & 42,9 & & 92,9 & & 0 & 7,1 & 92,9 & \\
\hline $15-19$ & 99,3 & & 55,3 & & 98,7 & & 8 & 20,7 & 71,3 & \\
\hline $20-34$ & 99,4 & 0,022 & 57,1 & 0,723 & 95,5 & 0,020 & 5,3 & 16,5 & 78,2 & 0,068 \\
\hline 35 ou mais & 100 & & 58,3 & & 94,4 & & 0 & 12,8 & 87,5 & \\
\hline \multicolumn{11}{|l|}{ Raça/cor da pele } \\
\hline Branca & 100 & & 60 & & 95,4 & & 4,6 & 13,8 & 81,7 & \\
\hline Preta & 100 & & 58,9 & & 98,2 & & 7,1 & 10,7 & 82,1 & \\
\hline Parda & 99,3 & 0,201 & 56,9 & 0,894 & 95,8 & 0,458 & 5,2 & 17,9 & 76,9 & 0,253 \\
\hline Amarela & 95,7 & & 47,8 & & 95,7 & & 0 & 17,4 & 82,6 & \\
\hline Indígena & 100 & & 66,7 & & 100 & & 33,3 & 33,3 & 33,3 & \\
\hline \multicolumn{11}{|l|}{ Escolaridade materna } \\
\hline Nenhuma & 100 & & 40 & & 100 & & 30 & 10 & 60 & \\
\hline Ensino Fundamental & 99,1 & & 55,8 & & 98,8 & & 7,5 & 21,1 & 71,4 & \\
\hline Ensino Médio & 99,4 & 0,912 & 61,9 & 0,025 & 97,4 & $<0,001$ & 3,2 & 16,5 & 80,3 & $<0,001$ \\
\hline Ensino Superior & 100 & & 45,5 & & 81,2 & & 1 & 4 & 95 & \\
\hline \multicolumn{11}{|l|}{ Situação conjugal } \\
\hline Sem companheiro & 99,5 & & 52 & & 96,2 & & 12,7 & 14,1 & 73,1 & \\
\hline Com companheiro & 99,4 & 0,630 & 58,6 & 0,016 & 97,9 & 0,009 & 5,6 & 18,1 & 76,3 & 0,691 \\
\hline \multicolumn{11}{|l|}{$\begin{array}{l}\text { Número de gestações } \\
\text { anteriores }\end{array}$} \\
\hline Nenhuma & 99 & & 59,1 & & 95,5 & & 2,7 & 15,5 & 81,8 & \\
\hline 1 & 100 & & 58,8 & & 96 & & 6 & 13,1 & 80,9 & \\
\hline 2 & 100 & 0,256 & 50,9 & 0,282 & 94,6 & 0,124 & 8 & 17 & 75 & 0,004 \\
\hline 3 ou mais & 98,3 & & 52 & & 98,4 & & 7,9 & 26 & 66,1 & \\
\hline \multicolumn{11}{|l|}{ Desejava engravidar } \\
\hline Queria engravidar & 100 & & 61,1 & & 96,5 & & 1,6 & 12 & 86,4 & \\
\hline Queria esperar mais & 100 & 0,023 & 52,5 & 0,032 & 95,6 & 0,099 & 8,8 & 18,2 & 72,9 & $<0,001$ \\
\hline Não queria engravidar & 98,1 & & 53,4 & & 95,5 & & 7,1 & 20,7 & 72,2 & \\
\hline \multicolumn{11}{|c|}{ Sentimentos quanto à gravidez } \\
\hline Satisfeita & 100 & & 60,4 & & 96,1 & & 3,7 & 13,8 & 82,5 & \\
\hline Mais ou menos satisfeita & 98 & 0,026 & 52 & 0,021 & 95,1 & 0,104 & 9,8 & 23,5 & 66,7 & $<0,001$ \\
\hline Insatisfeita & 98,6 & & 45,1 & & 97,2 & & 2,8 & 18,3 & 78,9 & \\
\hline \multicolumn{11}{|c|}{ Tentou interromper a gravidez } \\
\hline Não & 99,7 & & 57,9 & & 96,2 & & 4,9 & 16,3 & 78,7 & \\
\hline Sim & 92,1 & 0,001 & 34,2 & 0,008 & 92,1 & $<0,001$ & 10,5 & 26,3 & 62,2 & 0,221 \\
\hline Total & 99,3 & & 57 & & 96,6 & & *5,2 & ${ }^{\star} 16,9$ & ${ }^{\star} 74,7$ & \\
\hline
\end{tabular}


Tabela 2. Associações entre as orientações oferecidas às mulheres durante o pré-natal e o número de consultas realizadas $(\mathrm{n}=763)$. Sergipe, Brasil, 2015/2016.

\begin{tabular}{|c|c|c|c|c|}
\hline \multirow{3}{*}{ Orientações oferecidas às mulheres durante o pré-natal } & \multicolumn{2}{|c|}{ Número de consultas } & \multirow[t]{2}{*}{$\mathbf{p}$} & \multirow{2}{*}{$\begin{array}{c}\text { Total } \\
\mathrm{N}(\%) \\
\end{array}$} \\
\hline & $\leq 5(n=169)$ & $\geq 6(n=566)$ & & \\
\hline & $\mathbf{N}(\%)$ & $\mathbf{N}(\%)$ & & \\
\hline \multicolumn{5}{|l|}{ Atividades para facilitar o parto } \\
\hline Não & $116(25,2)$ & $345(74,8)$ & 0,051 & $461(62,6)$ \\
\hline Sim & $52(18,9)$ & $223(81,1)$ & & $275(37,4)$ \\
\hline \multicolumn{5}{|l|}{ Sinais de início do trabalho de parto } \\
\hline Não & $115(27,7)$ & $300(72,3)$ & 0,001 & $415(56,5)$ \\
\hline $\operatorname{Sim}$ & $54(16,9)$ & $266(83,1)$ & & $320(43,5)$ \\
\hline \multicolumn{5}{|l|}{ Alterações clínicas que indicariam risco da gravidez } \\
\hline Não & $92(29)$ & $225(71)$ & 0,001 & $317(43,10$ \\
\hline Sim & $77(18,4)$ & $342(81,6)$ & & $419(56,9)$ \\
\hline \multicolumn{5}{|l|}{ Importância da amamentação na primeira hora de vida } \\
\hline Não & $82(27,5)$ & $216(72,5)$ & 0,012 & $298(40,4)$ \\
\hline Sim & $86(19,6)$ & $353(80,4)$ & & $439(59,6)$ \\
\hline
\end{tabular}

\section{Discussão}

A cobertura da assistência pré-natal no Sergipe foi praticamente universal, com valores elevados em todos os municípios do estado, independentemente das características demográficas, sociais e reprodutivas das mulheres. Contudo, a adequação dessa assistência é ainda baixa às recomendações do $\mathrm{PHPN}^{4}$, pois $43 \%$ das entrevistadas iniciaram o pré-natal após a $16^{\text {a }}$ semana gestacional e $22,1 \%$ não tiveram o número mínimo de consultas previstas para a idade gestacional, o que indica busca tardia desta assistência e número insuficiente de atendimentos, situações também observadas em outros estudos nacionais realizados no Rio de Janeiro (RJ) com 2.422 gestantes (estudo transversal) $^{8}$, Juiz de Fora (MG) com 370 e 1.200 gestantes (estudo séries temporais múltiplas) ${ }^{18} e$, à nível nacional, em um estudo descritivo usando dados do SISPRENATAL ${ }^{19}$.

Ademais, ao comparar com os resultados do Nascer no Brasil ${ }^{9}$, inquérito nacional realizado com 23.894 mulheres e que também avaliou a adequação do acompanhamento pré-natal ao PHPN, observa-se um maior percentual de mulheres sergipanas com início tardio desta assistência. Sabe-se que idade precoce, menor escolaridade e dificuldade de acesso são alguns fatores associados a esta problemática, ${ }^{9,20}$.

Houve um padrão característico de mulheres com menor escolaridade, gravidez não planejada, insatisfeitas com a descoberta da gestação e que tentaram interrompê-la em apresentar menor cobertura pré-natal e/ou início mais tardio desta assistência. Estas características são também identificadas por outros estudos como fatores influenciadores do pré-natal ${ }^{9,20,21}$.

As gestantes adolescentes, principalmente as que possuíam idade $\leq 14$ anos, apresentaram menor cobertura da assistência pré-natal, resultado também já evidenciado ${ }^{9,20,22}$. Tal conduta pode estar relacionada ao desconhecimento sobre os serviços de pré-natal disponíveis, falta de autonomia decisória e estigmas sociais ${ }^{23}$, uma vez que a gestação na adolescência tem associação com estado civil não-casada ${ }^{24}$, demonstrando assim a necessidade de estratégias diferenciadas para esse grupo etário.

Gestantes consideradas de alto risco obstétrico apresentaram menor cobertura pré-natal, fato também discutido por Leal et al. ${ }^{25}$, que apontam a importância da identificação precoce destas mulheres como principal elemento na prevenção da morbimortalidade materna e infantil. Sabese que embora essas pacientes necessitem de um maior acesso e atenção do serviço de saúde, são justamente as que menos são vistas nas instituições responsáveis por elas.

A proporção de mulheres que receberam cartão de pré-natal durante seu acompanhamento foi elevada, sendo semelhante à encontrada na Pesquisa Nacional de Demografia e Saúde (PNDS) $2006^{26}$. A frequência destas que apresentaram o cartão de pré-natal na admissão para o 
Tabela 3. Distribuição proporcional das características maternas segundo a fonte de pagamento e o perfil da assistência prestada na maioria das consultas de pré-natal em uma amostra estadual de puérperas $(\mathrm{n}=763)$. Sergipe, Brasil, 2015/2016.

\begin{tabular}{|c|c|c|c|c|c|c|c|c|c|c|c|}
\hline \multirow[t]{2}{*}{$\begin{array}{l}\text { Exposição } \\
\text { Desfecho }\end{array}$} & \multicolumn{2}{|c|}{$\begin{array}{c}\text { Fonte de } \\
\text { pagamento }\end{array}$} & \multirow[t]{2}{*}{$\mathbf{p}$} & \multicolumn{2}{|c|}{$\begin{array}{c}\text { Tipo de unidade } \\
\text { de saúde }\end{array}$} & \multirow[t]{2}{*}{$\mathbf{p}$} & \multicolumn{2}{|c|}{$\begin{array}{c}\text { Categoria } \\
\text { profissional }\end{array}$} & \multicolumn{3}{|c|}{$\begin{array}{c}\text { Acompanhamento } \\
\text { pelo mesmo } \\
\text { profissional }\end{array}$} \\
\hline & Público & Privado & & $\begin{array}{c}\text { Atenção } \\
\text { Básica }\end{array}$ & $\begin{array}{c}\text { Ambulatório } \\
\text { hospitalar }\end{array}$ & & Médico & Enfermeiro & & $\%$ & \\
\hline Município & $\%$ & $\%$ & & $\%$ & $\%$ & & $\%$ & $\%$ & & & \\
\hline Aracaju & 65,3 & 33,6 & & 61,7 & 38,3 & & 59 & 41 & & 83,5 & \\
\hline Capela & 86,2 & 13,7 & & 65,5 & 34,5 & & 44,8 & 55,2 & & 86,2 & \\
\hline Estância & 93,5 & 6,4 & & 75,8 & 24,2 & & 22,6 & 77,4 & & 98,4 & \\
\hline Glória & 90,9 & 9 & & 81,8 & 18,2 & & 9,1 & 90,9 & & 86,4 & \\
\hline Itabaiana & 52,3 & 47,3 & $<0,001$ & 59,3 & 40,7 & 0,001 & 45,3 & 54,7 & $<0,001$ & 93 & $<0,001$ \\
\hline Lagarto & 80,3 & 19,7 & & 50 & 50 & & 47 & 53 & & 87,9 & \\
\hline Propriá & 91,4 & 8,6 & & 97,1 & 2,9 & & 14,3 & 85,7 & & 97,1 & \\
\hline Socorro & 95,8 & 4,2 & & 79,2 & 20,8 & & 45,8 & 54,2 & & 100 & \\
\hline \multicolumn{12}{|l|}{ Idade (anos) } \\
\hline $10-14$ & 78,6 & 21,4 & & 71,4 & 28,6 & & 50 & 50 & & 71,5 & \\
\hline $15-19$ & 84 & 16 & & 68,6 & 31,4 & & 38 & 62 & & 85,4 & \\
\hline $20-34$ & 68,8 & 31,2 & 0,001 & 63,7 & 36,3 & 0,050 & 50,4 & 49,6 & 0,010 & 89,1 & 0,029 \\
\hline 35 ou mais & 59,7 & 40,3 & & 59,7 & 40,3 & & 62,5 & 37,5 & & 83,3 & \\
\hline \multicolumn{12}{|l|}{ Raça/cor da pele } \\
\hline Branca & 59,6 & 40,4 & & 62,4 & 37,6 & & 52,3 & 47,7 & & 87,2 & \\
\hline Preta & 71,4 & 28,6 & & 71,4 & 28,6 & & 46,4 & 53,6 & & 83,9 & \\
\hline Parda & 73,1 & 26,9 & 0,273 & 63,5 & 36,5 & 0,159 & 48,8 & 51,2 & 0,979 & 87,7 & 0,124 \\
\hline Amarela & 73,9 & 26,1 & & 78,3 & 21,7 & & 47,8 & 52,2 & & 91,3 & \\
\hline Indígena & 100 & 0 & & 100 & 0 & & 33,3 & 66,7 & & 100 & \\
\hline \multicolumn{12}{|c|}{ Escolaridade materna } \\
\hline Nenhuma & 80 & 20 & & 90 & 10 & & 30 & 70 & & 80 & \\
\hline Fundamental & 88,2 & 11,8 & & 75,1 & 24,9 & & 35 & 65 & & 88,5 & \\
\hline Médio & 67,7 & 32,2 & $<0,001$ & 62,9 & 37,1 & $<0,001$ & 54,5 & 45,5 & $<0,001$ & 86,1 & 0,899 \\
\hline Superior & 21,8 & 78,2 & & 29,7 & 70,3 & & 83,2 & 16,8 & & 89,1 & \\
\hline \multicolumn{12}{|c|}{ Situação conjugal } \\
\hline $\begin{array}{l}\text { Sem } \\
\text { companheiro }\end{array}$ & 60,9 & 39,1 & & 47,5 & 52,5 & & 64,2 & 35,8 & & 83,4 & \\
\hline $\begin{array}{l}\text { Com } \\
\text { companheiro }\end{array}$ & 77,1 & 22,9 & $<0,001$ & 70,1 & 29,9 & $<0,001$ & 42,9 & 57,1 & 0,018 & 87,4 & 0,650 \\
\hline \multicolumn{12}{|c|}{ Número de gestações anteriores } \\
\hline Nenhuma & 68,2 & 31,8 & & 61,8 & 38,2 & & 51,5 & 48,5 & & 86,6 & \\
\hline 1 & 66,3 & 33,7 & & 62,3 & 37,7 & & 52,3 & 47,7 & & 89,4 & \\
\hline 2 & 75,9 & 24,1 & 0,053 & 67,9 & 32,1 & 0,154 & 49,1 & 50,9 & 0,050 & 87,5 & 0,412 \\
\hline 3 ou mais & 81,9 & 18,1 & & 71,7 & 28,3 & & 37,8 & 62,2 & & 86,6 & \\
\hline Total & *71,6 & *22 & & 64,9 & 35,1 & & *49,4 & *48 & & 88,1 & \\
\hline
\end{tabular}

Nota: $\%=$ Frequência relativa. ${ }^{\star}$ Foram excluídas da análise as participantes que não responderam integralmente às questões.

parto foi similar à observada em um estudo de âmbito nacional ${ }^{9}$, que também identificou percentuais reduzidos na internação para a parturição.

Apesar do registro do resultado da primeira glicemia ter sido elevado, não alcançou 100\% em nenhum dos municípios do estado. Ressalta-se que este exame deve ser solicitado a todas as gestantes na primeira consulta do pré-natal, como teste de rastreamento para o diabetes mellitus gestacional (DMG), independentemente da presença de fatores de risco, com interpretação se- 
Tabela 4. Distribuição proporcional das características maternas segundo o risco gestacional e a rede de assistência ao parto em uma amostra estadual de puérperas $(\mathrm{n}=763)$. Sergipe, Brasil, 2015/2016.

\begin{tabular}{|c|c|c|c|c|c|c|c|c|c|c|}
\hline \multirow[t]{2}{*}{$\begin{array}{l}\text { Exposição/ } \\
\text { Desfecho }\end{array}$} & \multirow{2}{*}{$\begin{array}{c}\begin{array}{c}\text { Gestação } \\
\text { de risco }\end{array} \\
\%\end{array}$} & \multirow[t]{2}{*}{ p } & \multicolumn{3}{|c|}{$\begin{array}{c}\text { Atendida em serviço de } \\
\text { referência }\end{array}$} & \multirow[t]{2}{*}{$\mathbf{p}$} & \multirow{2}{*}{$\begin{array}{c}\begin{array}{c}\text { Orientada } \\
\text { sobre }\end{array} \\
\text { maternidade } \\
\text { de referência }\end{array}$} & \multirow{2}{*}{$\mathbf{p}$} & \multirow{2}{*}{$\begin{array}{c}\begin{array}{c}\text { Peregrinação } \\
\text { para o parto }\end{array} \\
\%\end{array}$} & \multirow[t]{2}{*}{$\mathbf{p}$} \\
\hline & & & Não & $\begin{array}{c}\text { Sim, com } \\
\text { dificuldade }\end{array}$ & $\begin{array}{c}\text { Sim, sem } \\
\text { dificuldade }\end{array}$ & & & & & \\
\hline Município & & & $\%$ & $\%$ & $\%$ & & & & & \\
\hline Aracaju & 22,1 & & 9,1 & 25,5 & 65,5 & & 66,4 & & 30,6 & \\
\hline Capela & 17,2 & & 0 & 33,3 & 66,7 & & 58,6 & & 41,4 & \\
\hline Estância & 6,5 & & 0 & 25 & 75 & & 38,7 & & 37,1 & \\
\hline Glória & 9,1 & 0,001 & 100 & 0 & 0 & 0,049 & 45,5 & 0,070 & 22,7 & 0,001 \\
\hline Itabaiana & 4,7 & & 0 & 0 & 100 & & 61,6 & & 22,1 & \\
\hline Lagarto & 15,2 & & 0 & 0 & 100 & & 59,1 & & 40,9 & \\
\hline Propriá & 11,4 & & 100 & 0 & 0 & & 60 & & 5,7 & \\
\hline Socorro & 12,5 & & 50 & 0 & 50 & & 54,2 & & 8,3 & \\
\hline \multicolumn{11}{|l|}{ Idade (anos) } \\
\hline $10-14$ & 57,1 & & 20 & 20 & 60 & & 64,3 & & 35,7 & \\
\hline $15-19$ & 19,3 & $<0,001$ & 6,2 & 31,2 & 62,5 & 0,591 & 51,3 & 0,003 & 42 & 0,001 \\
\hline $20-34$ & 13,2 & & 16,7 & 19,4 & 63,9 & & 61,5 & & 27,1 & \\
\hline 35 ou mais & 31,9 & & 0 & 20 & 80 & & 81,9 & & 19,4 & \\
\hline \multicolumn{11}{|l|}{ Raça/cor da pele } \\
\hline Branca & 11 & & 16,7 & 16,7 & 66,6 & & 67 & & 26,6 & \\
\hline Preta & 16,1 & & 0 & 0 & 100 & & 60,7 & & 17,9 & \\
\hline Parda & 18,1 & 0,428 & 10,5 & 24,6 & 64,9 & 0,489 & 60,6 & 0,941 & 30,6 & 0,115 \\
\hline Amarela & 17,4 & & 33,3 & 33,3 & 33,3 & & 60,9 & & 39,1 & \\
\hline Indígena & 33,3 & & 0 & 0 & 100 & & 33,3 & & 66,7 & \\
\hline \multicolumn{11}{|c|}{ Escolaridade materna } \\
\hline Nenhuma & 30 & & 50 & 0 & 50 & & 60 & & 40 & \\
\hline Fundamental & 19,7 & & 10,8 & 21,6 & 67,6 & & 53,5 & & 38,7 & \\
\hline Médio & 14,8 & 0,250 & 7,7 & 30,8 & 61,5 & 0,370 & 63,9 & $<0,001$ & 25,5 & $<0,001$ \\
\hline Superior & 12,9 & & 14,3 & 0 & 85,7 & & 82,2 & & 8,9 & \\
\hline \multicolumn{11}{|l|}{ Situação conjugal } \\
\hline $\begin{array}{l}\text { Sem } \\
\text { companheiro }\end{array}$ & 23,1 & & 6,9 & 20,2 & 72,9 & & 67,2 & & 26,5 & \\
\hline $\begin{array}{l}\text { Com } \\
\text { companheiro }\end{array}$ & 16,9 & 0,679 & 12,2 & 17,1 & 70,7 & 0,758 & 59,2 & $<0,001$ & 31,1 & 0,174 \\
\hline \multicolumn{11}{|c|}{ Número de gestações anteriores } \\
\hline Nenhuma & 16,1 & & 13,3 & 10 & 76,7 & & 60,6 & & 32,1 & \\
\hline 1 & 14,1 & & 5,3 & 36,8 & 57,9 & & 61,3 & & 29,1 & \\
\hline 2 & 20,5 & 0,320 & 20 & 20 & 60 & 0,427 & 59,8 & 0,389 & 23,2 & 0,346 \\
\hline 3 ou mais & 20,5 & & 11,1 & 27,8 & 61,1 & & 65,4 & & 28,3 & \\
\hline Total & 16,6 & & 11,4 & 21,4 & 67,2 & & 61,3 & & 29,4 & \\
\hline
\end{tabular}

gundo esquema disponibilizado pelo Ministério da Saúde ${ }^{2}$. Foi detectada também elevada cobertura de exames de ultrassonografia, cuja realização torna-se importante ao revelar o estado de saúde do bebê, reduzir as preocupações dos pais e promover a construção da própria maternidade ${ }^{27}$.
A baixa proporção de orientações oferecidas às mulheres durante a assistência pré-natal em Sergipe evidencia o despreparo dos profissionais de saúde que atuam na preparação das mulheres para o parto e para a amamentação, sendo observada apenas a priorização de orientação sobre 


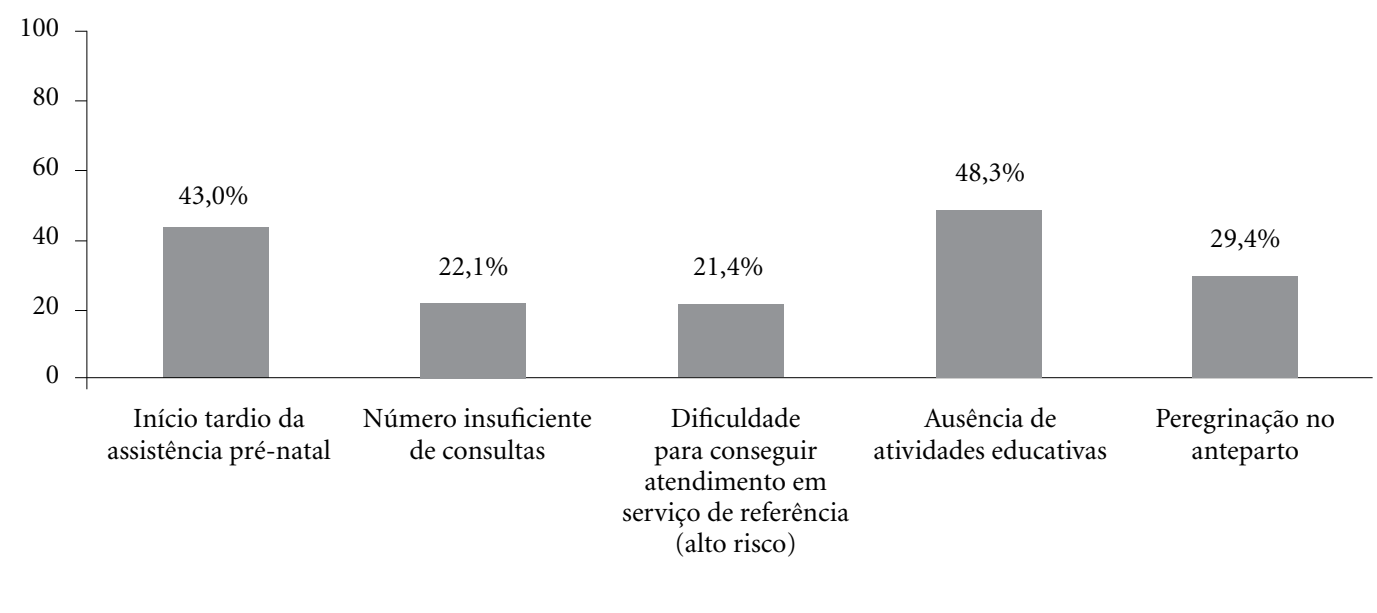

Gráfico 1. Principais inadequações da assistência pré-natal em Sergipe em relação às recomendações do PHPN ( $\mathrm{n}=763$ para todas as variáveis, exceto "Dificuldade no atendimento em serviço de referência" cuja amostra é 70). Sergipe, Brasil, 2015/2016.

sinais de risco na gravidez. Esse tipo de prioridade é justificado pelo caráter biomédico da assistência à saúde. Nesse sentido, espera-se que, no âmbito da interdisciplinaridade, o conhecimento biomédico esteja mais articulado às outras ciên$\operatorname{cias}^{28}$.

Quanto à organização da assistência prénatal, observa-se predominância de utilização dos serviços públicos, sobretudo de unidades da atenção básica. Apenas 35,1\% das entrevistadas foram acompanhadas em unidades hospitalares. Isto parece adequado, pois somente as pacientes com maior risco devem ser seguidas em unidades de maior nível de complexidade. Estudo de abrangência nacional também mostrou maior prevalência de pré-natal em serviços públicos?.

Percebeu-se que a assistência pré-natal no estado de Sergipe é realizada na mesma frequência por ambas categorias profissionais, médica e de enfermagem. Este resultado corrobora com um estudo nacional ${ }^{9}$ que identificou atuação equivalente destes profissionais nas regiões Norte e Nordeste do país. Vale ressaltar que as consultas de pré-natal e puerpério podem ser realizadas pelo profissional médico ou de enfermagem, quando de baixo risco ${ }^{2}$.

A maioria das puérperas $(88,1 \%)$ referiu acompanhamento pelo mesmo profissional durante a assistência pré-natal, indicando a continuidade desta atenção. Isto, torna-se fundamental para a criação de vínculos e relação de confiança entre o profissional e a gestante, bem como permite melhor monitoramento da gestação?.

Quanto à articulação da assistência pré-natal com outros serviços da rede de saúde do estado, evidenciou-se relatos de problemas pelas gestantes de alto risco encaminhadas para os serviços de referência. Vale ainda ressaltar que quase metade $(45,7 \%)$ das mulheres de alto risco obstétrico continuaram seu acompanhamento pré-natal com profissionais enfermeiros. Isso caracteriza problemas na organização desta assistência, já que gestantes de risco são as que mais se beneficiariam de um atendimento especializado e, portanto, cabe ao enfermeiro encaminhá-las ao profissional médico ${ }^{2}$.

Outra falha da assistência pré-natal está relacionada à orientação da gestante sobre a maternidade de referência, que foi relatada por apenas $61,3 \%$ das entrevistadas. A peregrinação pela busca de assistência ao parto também foi elevada $(29,4 \%)$, sendo superior à encontrada na PNDS $2006^{26}$, principalmente entre mulheres com menor idade e escolaridade. Sabe-se que tal achado acarreta riscos para a saúde da mulher e do bebê, pois de acordo com Menezes et al. ${ }^{29}$, estas mulheres buscam assistência em outro estabelecimento pelos próprios meios. Em seu estudo, realizado na cidade do Rio de Janeiro com 6.652 puérperas, apenas $1 / 5$ das mulheres foi transportada por ambulância. 
Neste contexto de risco para a saúde materno-infantil, vale ressaltar que a região Nordeste, entre 2000 e 2009, apresentou os maiores índices de mortalidade materna do país ${ }^{30}$. Isso mostra a necessidade de intervenções no período gravídico -puerperal, sobretudo voltadas à prevenção, para que se obtenha indicadores satisfatórios nessa área.

As limitações deste estudo estão relacionadas à confiabilidade dos dados obtidos por meio do relato das puérperas entrevistadas, tais como as orientações oferecidas pelos profissionais durante o pré-natal, a peregrinação no anteparto e a dificuldade para conseguir atendimento nos serviços de referência para alto risco.

Esse estudo permitiu afirmar que Sergipe atingiu uma boa cobertura da assistência prénatal, alcançando praticamente a totalidade das gestantes do estado. Entretanto, há vários dados que indicam ser de qualidade e adequação insuficientes ao PHPN. Os principais problemas identificados foram: início tardio do pré-natal; número insuficiente de consultas; poucas orientações, inclusive sobre a maternidade de referência para o parto; peregrinação no anteparto; falta de priorização das gestantes de maior risco e problemas na articulação com outros serviços de saúde materno-infantil. Ressalta-se que a redução da morbimortalidade materno-infantil requer o início precoce do acompanhamento pré-natal e a utilização dos recursos preconizados pelo Ministério da Saúde, desde ações de promoção e prevenção da saúde até o diagnóstico e tratamento adequado dos problemas que possam vir a ocorrer no período gravídico-puerperal. 


\section{Colaboradores}

RB Mendes: contribuiu substancialmente para a concepção e planejamento, e análise e interpretação dos dados; contribuiu significativamente na elaboração do rascunho e na revisão crítica do conteúdo; e participou da aprovação da versão final do manuscrito. JMJ Santos: contribuiu substancialmente para a concepção e planejamento, $\mathrm{e}$ análise e interpretação dos dados; contribuiu significativamente na elaboração do rascunho e na revisão crítica do conteúdo; e participou da aprovação da versão final do manuscrito. RQ Gurgel: contribuiu substancialmente para a concepção e planejamento, e análise e interpretação dos dados; contribuiu significativamente na elaboração do rascunho e na revisão crítica do conteúdo; e participou da aprovação da versão final do manuscrito. DS Prado: contribuiu significativamente na elaboração do rascunho e na revisão crítica do conteúdo; e participou da aprovação da versão final do manuscrito. RQ Gurgel: contribuiu significativamente na elaboração do rascunho e na revisão crítica do conteúdo; e participou da aprovação da versão final do manuscrito. FD Bezerra: contribuiu significativamente na elaboração do rascunho e na revisão crítica do conteúdo; e participou da aprovação da versão final do manuscrito.

\section{Referências}

1. Brasil. Ministério da Saúde (MS). Atenção ao pré-natal de baixo risco. Brasília: MS; 2012. (Caderno de Atenção Básica no 32).

2. Brasil. Ministério da Saúde (MS). Pré-natal e Puerpério: atenção qualificada e humanizada - manual técnico. Brasília: MS; 2005. (Série A, Caderno n 5).

3. Carroli G, Rooney C, Villar J. How effective is antenatal care in preventing maternal mortality and serious morbidity? An overview of the evidence. Paediatr Perinat Epidemiol 2001; 15(Supl. 1):1-42.

4. Brasil. Ministério da Saúde (MS). Portaria $n^{\circ} 569$, de $1^{\circ}$ de junho de 2000. Institui o Programa de Humanização no Pré-natal e Nascimento (PHPN). Diário Oficial da União; 2000.

5. Pessoa IN, Menezes ED, Ferreira TF, Dotto LMG, Bessa LF. Percepção de puérperas sobre assistência de enfermagem na gravidez. Cien Cuid Saude 2009; 8(2):236-241.

6. Coimbra LC, Silva AAM, Mochel EG, Alves MTSSB, Ribeiro VS, Aragão VMF, Bettiol H. Fatores associados à inadequação do uso da assistência pré-natal. Rev Saude Publica 2003; 37(4):456-462.

7. Carvalho DS, Novaes HMD. Avaliação da implantação de programa de atenção pré-natal no Município de Curitiba, Paraná, Brasil: estudo em coorte de primigestas. Cad Saude Publica 2004; 20(Supl. 2):S220-S230.

8. Domingues RMSM, Hartz ZMDA, Dias MAB, Leal MDC. Avaliação da adequação da assistência pré-natal na rede SUS do Município do Rio de Janeiro, Brasil. Cad Saude Publica 2012; 28(3):425-437.

9. Viellas EF, Augusto M, Dias B, Viana J, Bastos MH. Assistência pré-natal no Brasil. Cad Saude Publica 2014; 30(Supl. 1):S85-S100.

10. Brasil. MInistério da Saúde (MS). Departamento de Informática do SUS (DATASUS) [página na Internet]. 2014 [acessado 2017 Maio 21]. Disponível em: http:// www2.datasus.gov.br/DATASUS/index.php

11. Andreucci CBCB, Cecatti JGJG. Desempenho de indicadores de processo do Programa de Humanização do Pré-natal e Nascimento no Brasil: uma revisão sistemática. Cad Saude Publica 2011; 27(6):1053-1064.

12. Leal MC, coordenadora. Cadernos de Saúde Pública. Suplemento especial sobre a Pesquisa Nascer no Brasil. Rio de Janeiro: ENSP/Fiocruz; 2014.

13. Leal MC, Silva AAM, Dias MAB, Gama SGN, Rattner D, Moreira ME, Theme Filha MM, Domingues RMSM, Pereira APE, Torres JÁ, Bittencourt DAS, d'Orsi E, Cunha AJLA, Leite AJM, Cavalcante RS, Lansky S, Diniz CSG, Szwarcwald CL. Birth in Brazil: national survey into labour and birth. Reprod Health 2012; 9:15.

14. Santos Neto ET, Leal MC, Oliveira AE, Zandonade E, Gama SGN. Concordância entre informações do Cartão da Gestante e da memória materna sobre assistência pré-natal. Cad Saude Publica 2012; 28(2):256-266.

15. World Health Organization (WHO). Problemas de la salud de la adolescência. Informe de un comité de expertos de la OMS. Genebra: WHO; 1965.

16. Brasil. Ministério da Saúde (MS). Conselho Nacional de Saúde. Resolução no 466, de 12 de dezembro de 2012. Diário Oficial da União 2013; 13 dez. 
17. Brasil. Ministério da Saúde (MS). Conselho Nacional de Saúde. Resolução no 196 de 10 de outubro de 1996. Diretrizes e Normas Regulamentadoras de Pesquisas Envolvendo Seres Humanos. Diário Oficial da União 1996; 16 out.

18. Coutinho T, Monteiro MFG, Sayd JD, Teixeira MTB, Coutinho CM, Coutinho LM. Monitoramento do processo de assistência pré-natal entre as usuárias do Sistema Único de Saúde em município do Sudeste brasileiro. Rev Bras Ginecol Obs 2010; 32(11):563-569.

19. Serruya SJ, Cecatti JG, Lago TDG. O Programa de Humanização no Pré-natal e Nascimento do Ministério da Saúde no Brasil: resultados iniciais. Cad Saude Publica 2004; 20(5):1281-1289.

20. Pereira PHG, Antón AGS, Vieira Junior WS, Domingues RAD, Melo AL, Farias CS, Paula TF, Carvalho RP, Araújo PR, Godoy RB, Muza GM. Fatores associados ao acesso tardio ao pré-natal do Centro de Saúde $n^{\circ} 1$ do Paranoá, 2005. Comun Cien Saude 2006; 17(2):101-110.

21. Bassani DG, Surkan PJ, Olinto MTA. Inadequate use of prenatal services among Brazilian women: The role of maternal characteristics. Int Perspect Sex Reprod Health 2009; 35(1):15-20.

22. Wiemann CM, Berenson AB, Pino LG, McCombs SL. Factors associated with adolescents' risk for late entry into prenatal care. Fam Plann Perspect 1997; 29(6):273-276.

23. Miranda FRD, Taquette SR, Monteiro DLM, Blanco MN, Rodrigues AO. Pré-natal na adolescência: uma revisão crítica. Adolesc Saude 2013; 10(1):43-50.

24. Lessa FS, Cunha AA, Pinhal IMC, Bornia RG, Nejaim JE. A adolescência como fator de risco social na gravidez. Adolesc Saude 2006; 3(2):29-32.
25. Leal MC, Gama SGN, Ratto KMN, Cunha CB. Uso do índice de Kotelchuck modificado na avaliação da assistência pré-natal e sua relação com as características maternas e o peso do recém-nascido no Município do Rio de Janeiro. Cad Saude Publica 2004; 20(1):63-72.

26. Brasil. MInistério da Saúde (MS). Pesquisa nacional de demografia e saúde da criança e da mulher: PNDS2006: dimensões do processo reprodutivo e da saúde da criança. Brasília: MS; 2009.

27. Piccinini CA, Carvalho FT, Ourique LR, Lopes RS. Percepções e sentimentos de gestantes sobre o pré-natal. Psic Teor e Pesq 2012; 28(1):27-33.

28. Líbera BD, Saunders C, Santos MMAS, Rimes KA, Brito FRSS, Baião MR. Avaliação da assistência prénatal na perspectiva de puérperas e profissionais de saúde. Cien Saude Colet 2011; 16(12):4855-4864.

29. Menezes DCS, Leite IC, Schramm JMA, Leal MC. Avaliação da peregrinação anteparto numa amostra de puérperas no Município do Rio de Janeiro, Brasil, 1999/2001. Cad Saude Publica 2006; 22(3):553-559.

30. Ferraz L, Bordignon M. Mortalidade materna no brasil: uma realidade que precisa melhorar. Rev Baiana Saude Publica 2012; 36(2):527-538.

Artigo apresentado em 24/11/2017

Aprovado em 18/07/2018

Versão final apresentada em 20/07/2018 